\title{
Diagnosis, Prevention and Treatment of White Spot Lesions Related to Orthodontics
}

\author{
Sitkı Sarper Temel ${ }^{1}$ and Burçak Kaya ${ }^{2 *}$ \\ ${ }^{1}$ PhD Student, Faculty of Dentistry, Department of Orthodontics, Baskent University, Turkey \\ ${ }^{2}$ Associate Professor, Faculty of Dentistry, Department of Orthodontics, Baskent University, Turkey
}

*Corresponding author: Dr. Burçak Kaya, Associate Professor, Faculty of Dentistry, Department of Orthodontics, Baskent University, 1. Cad No: 107, 06490 Bahcelievler-Ankara, Turkey, Tel: +90-312-203-00-15, Fax: +90-312-203-00-26

\begin{abstract}
The purpose of this review article is to examine the etiology, incidence and classification of white spot lesions in association with their prevention and treatment methods. White spot lesions are opacities that occur by demineralization of enamel under the surface and cause esthetic problems. Orthodontic treatment increases the risk of white spot lesion occurrence by creating areas difficult to clean and prone to plaque accumulation around orthodontic attachments. Therefore, application of suitable methods for prevention of these lesions during orthodontic treatment and performing appropriate therapies after orthodontic treatment is important for obtaining favorable results and patient satisfaction. Oral hygiene motivation, usage of topical fluoride agents, casein phosphopeptide-amorf calcium phosphate agents, antimicrobial agents, tooth bleaching, microabrasion and resin infiltration are current techniques that clinicians can use for prevention and treatment of white enamel lesions.
\end{abstract}

\section{Keywords}

White spot lesion, Enamel demineralization, Plaque accumulation, Oral hygiene, Fluoride, CPP-ACP, Resin infiltration, Orthodontic treatment

\section{Introduction}

Enamel decalcification occurs when bacterial flora remains on enamel surface for a long time [1]. Organic acids produced by bacteria enter interprismatic spaces in tooth enamel, resulting in white lesions due to dissolution of apatite crystals, calcium and phosphate ions, and demineralization [2].

Generally, enamel discoloration can be seen in the form of dental fluorosis, opacity and white spot lesions.
Fluorosis is a white to yellowish colored lesion that is not completely distinguishable, fused with normal enamel and symmetrically distributed. On the other hand, opacities that are not fluoride-related have more specific shapes, differ from tooth enamel and are often found in the middle of teeth. In patients receiving orthodontic treatment, white spot lesions often occur beneath broken bands, around bracket bases, and in regions where brushing is difficult [3]. The purpose of this review article is to examine the etiology, incidence and classification of white spot lesions in association with their prevention and treatment methods.

\section{Methods}

The search strategy was organized across 4 electronic databases (Pubmed, Web of Science, Medline, Scopus) using search terms such as "orthodontic treatment", "white spot", "lesion", "white discoloration", "CPP-ACP" and "Fluoride". Abstracts for all relevant terms were retrieved and evaluated independently by 2 reviewers (S.S.T. and B.K.). Exclusion criteria were developed for study selection. Studies published before 1975 and not available in full text in English were excluded. Data selection was completed by one reviewer (S.S.T.) and checked for accuracy and consistency by a second reviewer (B.K.). Mendeley reference management software was used for organization of citations.

\section{Relationship of white spot lesions with orthodon- tics}

Adhesives around orthodontic attachments constitute areas that are suitable for increase in bacterial

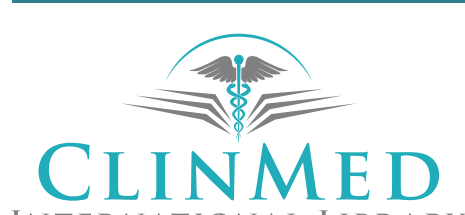

INTERNATIONAL LIBRARY
Citation: Temel SS, Kaya B (2019) Diagnosis, Prevention and Treatment of White Spot Lesions Related to Orthodontics. Int J Oral Dent Health 5:085. doi.org/10.23937/2469-5734/1510085

Accepted: April 03, 2019: Published: April 05, 2019

Copyright: (c) 2019 Temel SS, et al. This is an open-access article distributed under the terms of the Creative Commons Attribution License, which permits unrestricted use, distribution, and reproduction in any medium, provided the original author and source are credited. 

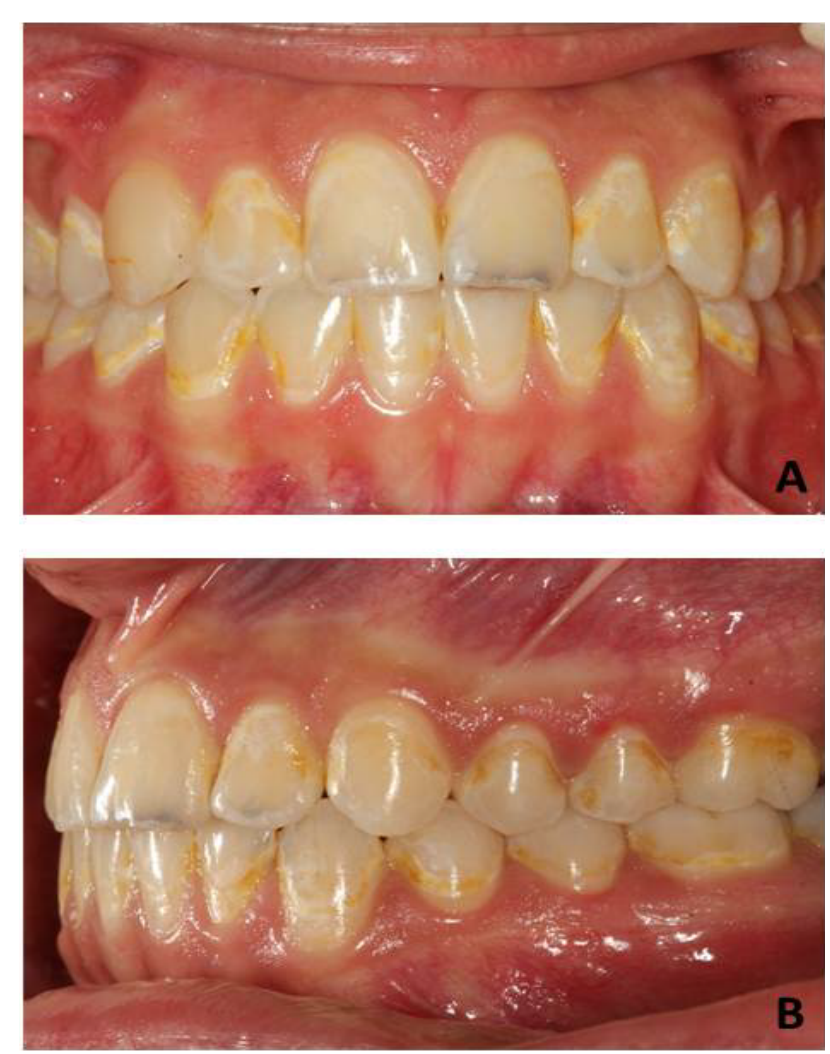

Figure 1: A patient presenting mild to severe white spot lesions in number of teeth after orthodontic treatment. A) Intraoral frontal view; B) Intraoral lateral view.

counts [4]. It was reported that following application of orthodontic appliances, a rapid change in bacterial composition of plaque was observed, and the amount of acidogenic bacteria such as Streptococcus mutans and Lactobacillus significantly increased. These bacteria reduce $\mathrm{pH}$ level of plaque in presence of fermentable carbohydrates. Decalcification occurs when the $\mathrm{pH}$ level of oral environment falls below the threshold level of remineralization [3].

Irregular composite residues around brackets increase plaque accumulation, but fluoride-containing or anti-microbial composites decrease the acidity level of oral environment and prevent demineralization. In general, white lesions due to orthodontic treatment occur on buccal surface of teeth and in areas that are difficult to clean (Figure 1). These are the areas between brackets and gingiva, especially in upper lateral and canine teeth and lower canine and first premolar teeth [1].

Gorelick, et al. [1] reported the factors affecting formation of white spot lesions as; surface characteristics of teeth, saliva access and distance between brackets and gingiva. No association between white lesion formation and lingual retainers was observed. This was explained with the protective role of saliva flow amount and buffering capacity against acid attacks.

\section{Incidence of white spot lesions}

Despite there are differences between the studies, the most affected teeth examined are upper lateral incisors, upper canines, lower canines and lower first molars, respectively [1].

\section{Classification of white spot lesions}

White-spot lesions were first classified in orthodontics according to their visual size [5]. This classification made according to the width of opacity formed on enamel surface was as follows:

Class 0: None or less than $1 \mathrm{~mm}$ opacity

Class 1: Opacity covers $1 / 3$ of tooth surface

Class 2: Opacity covers $1 / 3$ to $2 / 3$ of tooth surface

Class 3: Opacity covers wider than $2 / 3$ of tooth surface

Another classification made by Gorelick, et al. [1], which considers both size and intensity of lesions is as follows:

Class 1: No white spot lesion formation

Class 2: Mild white spot lesion present

Class 3: Severe white spot lesion present

Class 4: Cavitation is present in addition to white spot lesion

\section{Evaluation of white spot lesions}

Accurate and rapid evaluation of white spot lesions during orthodontic treatment is very important for performing protective, preventive and corrective therapies [6]. Assessment of white spot lesions requires two steps. The first step involves evaluation of decalcification and the second step involves evaluation of severity of a lesion. Severity is assessed according to the brightness and size of a discoloration macroscopically, whereas according to the amount of mineral loss and lesion depth microscopically [6].

\section{Prevention of white spot lesions}

The structure of hydroxyapatite crystals is under the influence of a natural cycle between demineralization and remineralization. This cycle works in the favor of either demineralization or remineralization, depending on environmental factors [7]. Studies using polarized light microscopy indicate that the size of white lesions may decreases over time [8]. There are studies showing that white lesions resulting from orthodontic treatment decrease rapidly after removal of orthodontic appliances within 12 months and may decrease up to $50 \%$ within 24 months [8]. The degree of remineralization varies between individuals and different regions within the mouth. Sometimes the size of a demineralization area may be such that it cannot be improved even if an effective remineralization agent is used. Therefore, methods to prevent white lesion formation is important [8].

During orthodontic treatment, the best method is to 
prevent white spot lesions before they develop. There are two fundamental methods to accomplish this. The first one is to avoid breaking of ongoing demineralization or to strengthen remineralization process. The second one is to prevent demineralization on tooth surface [9].

Methods for increasing remineralization and decreasing demineralization in patients undergoing orthodontic treatment are; oral hygiene motivation, regular professional oral hygiene appointments, usage of topical agents and orthodontic adhesives involving fluoride [9].

Saliva is also very important information or prevention of tooth decay. Degradation of minerals in enamel after acid attacks, demineralization degree, the onset or duration of remineralization are related to the $\mathrm{pH}$ level of saliva [10].

Oral hygiene motivation: The $\mathrm{pH}$ level around braces can easily decrease in patients receiving orthodontic treatment. If patient has good oral hygiene, $\mathrm{pH}$ level will not exceed the critical level in early stages of acid attacks. However, if patient's oral hygiene is poor, permanent mineral loss can be observed around braces, as these areas remain below critical $\mathrm{pH}$ level for a long time.

Reducing the amount of plaque on tooth surface is an effective method for preventing caries formation [11]. Removal of dental plaque is possible by mechanical and chemical methods. Tooth brushing is a commonly used method for mechanically controlling plaque. Mouthwashes involving different ingredients that provide chemical plaque control are also effective agents that reduce bacterial count by $99.9 \%$ without damaging the surrounding oral tissues. Mouthwashes containing chlorhexidine are the most effective [12].

Usage of agents with fluoride: Fluoride application is a commonly used method to reduce enamel tendency to demineralization. The concentration of fluoride in saliva and plaque is effective in prevention of demineralization and formation of remineralization [13]. The organic acids formed by cariogenic bacteria cause decrease in $\mathrm{pH}$ level of plaque, which results in fluoride diffusion into enamel from plaque and saliva in response. Displacement of hydroxyl ions of enamel structure with fluoride causes existence of fluorapatite crystals. This new crystal form is more resistant to acids [14]. Fluoride also affects the activities of cariogenic bacteria and prevents formation of caries. Laboratory studies have shown that low concentrations of fluoride cause Streptococcus mutans to produce less amount of acid [15].

The concentration of fluoride in enamel surface decreases dramatically with depth. Fluoride has low solubility and tends to accumulate on enamel surface. It cannot go deeper in enamel layers after porous cavities are filled with it. In a study, fluoride was applied for 3 months to a white lesion of $100 \mu \mathrm{m}$ depth which was created in experimental conditions. Following 3 months of application, fluoride ions could only reach a depth of $50 \mu \mathrm{m}$ [16]. Therefore, a low dose of fluoride is recommended for the fluoride ions to reach deeper layers of lesions easily. Lee Linton [17] claims that a mouthwash containing $50 \mathrm{ppm}$ fluoride is more effective in remineralization than one that contains $250 \mathrm{ppm}$ fluoride. On the other hand, according to the results of a clinical study published by Willmot, et al. [18], there is no additional remineralization effect of mouthwashes and toothpastes with low dose fluoride compared with the fluoride-free ones. Additionally, it is reported that the ability of fluoride ion to remineralize is less than its ability to prevent demineralization.

Discolorations due to fluorosis are caused by excessive use of fluoride during development of enamel [19]. High levels of fluoride in drinking water, toothpastes, nutritional supplements and dental materials are risk factors for fluorosis, particularly in children under 8 years of age [20].

It has been reported that the best results in fluoride administration can be taken from daily use of low concentration agents. Although enamel may dissolve in low $\mathrm{pH}$ levels, presence of low concentration of fluoride provides the dissolved minerals to participate in remineralization cycle and prevent mineral loss [21]. There is a critical $\mathrm{pH}$ level for fluoride to be effective in formation of fluorapatite. The $\mathrm{pH}$ levels between which fluoride works more effectively are reported as 4.5-6. The working mechanism of fluoride in accordance with $\mathrm{pH}$ level can be explained by the Stephan Curve (Figure 2).

Usage of agents with casein phosphopeptideamorf calcium phosphate (CPP-ACP): Harper, et al. [22] compared the anticariogenic potentials of four different type of cheese, each containing different levels of fat, protein, calcium and phosphate. It was found that cheese with the most protective properties against caries formation was the one that involves the greatest amount of casein phosphoproteins and calcium phosphate in its content.

Acid casein, which is a protein commonly found in mammalian milk, can reduce formation of caries when added into toothpastes. However, the amount of casein required makes any toothpaste unusable because of its taste. Sodium caseinate added into chocolate also reduces cariogenicity, but it is stated that the amount of caseinate required (16.6\%) makes the product unusable for the same reason [23].

The anticariogenic mechanism of CPP-ACP can be summarized as increasing the level of calcium phosphate in plaque, reducing demineralization and increasing remineralization of enamel [23]. There are studies showing that CPP inhibits adherence and functioning of cariogenic streptococcus bacteria in mouth [23]. 


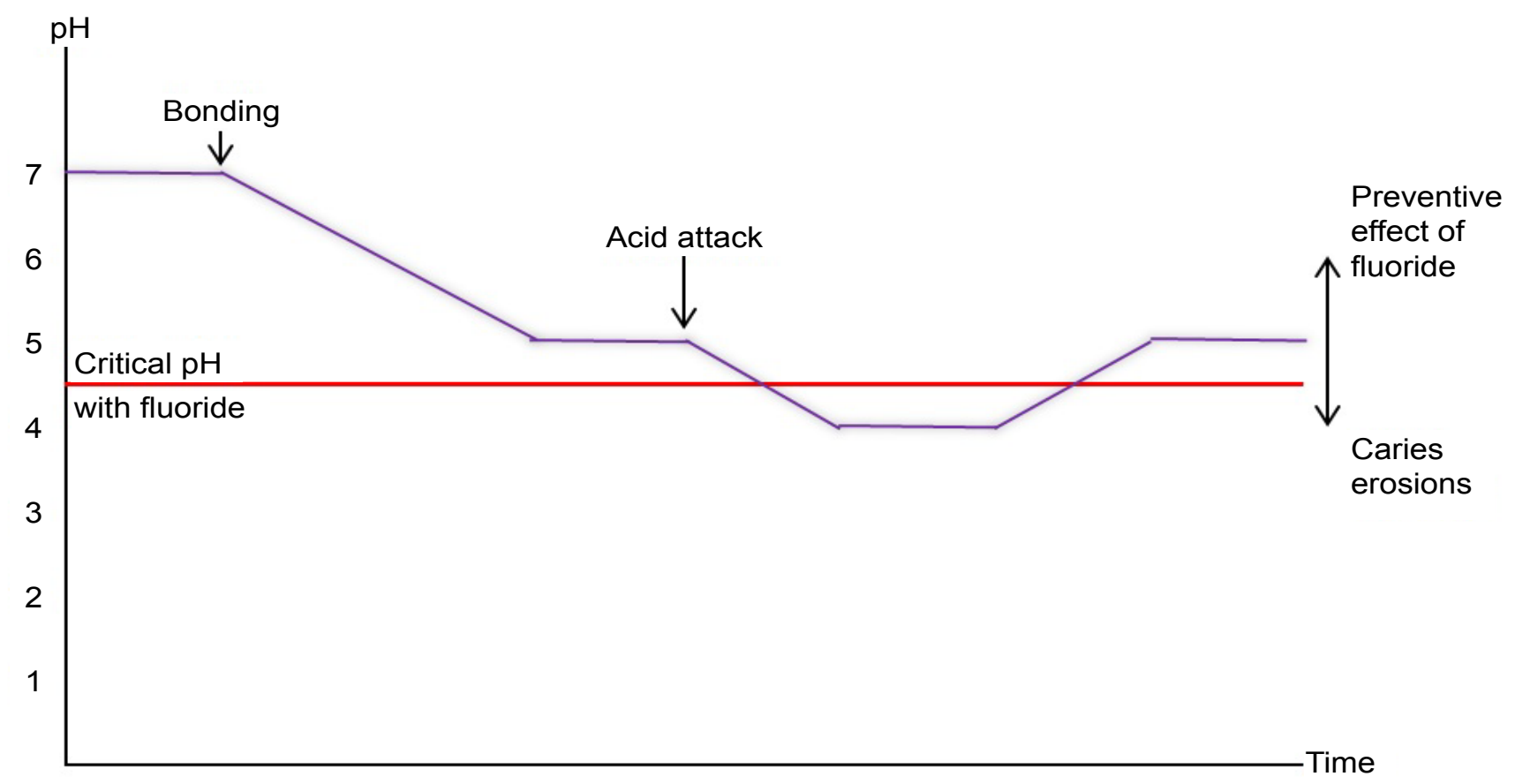

Figure 2: Stephan curve showing the working mechanism of fluoride in accordance with pH level.

Scupbach, et al. [24] showed that the adherence ability of bacteria such as Streptococcus sobrinus and Streptococcus mutans decreased considerably after administration of CPP. Reynolds, et al. [25] showed that use of CPP-ACP between $0.5-1.0 \%$ concentration is equivalent to $500 \mathrm{ppm}$ of fluoride in reducing cariogenic activity. An advantage of CPP-ACP over fluoride is not causing fluorosis due to high doses. Therefore use of CPP-ACP may reduce the risk of fluorosis as it reduces the need for fluoride [26].

It was thought that remineralization capacity of fluoride ions could be increased by using calcium ions. Nevertheless, the success of remineralization obtained by calcium fluoride which was formed when fluoride and calcium ions came together was found to be inadequate [27].

Usage of antimicrobial agents: Chemotherapeutic agents cannot prevent formation of dental plaque, but they can be used to remove microorganisms, which is one of the main factors that cause enamel demineralization. Chlorhexidine and benzalkonium chloride are the most preferred antimicrobial agents for this purpose [28]. There are a number of studies showing that these agents significantly reduce Streptococcus mutans levels, which are responsible for demineralization and caries formation $[28,29]$.

Regular use of these agents twice a day causes discoloration on teeth. Therefore it has been reported that $0.2 \%$ chlorhexidine mouthwash can be used at certain intervals in addition to other protection methods during orthodontic treatment, in order to reduce its side effects [30].

Usage of xylitol: It is known that xylitol shows anticariogenic effect by limiting caries lesion. This effect is based on the fact that it is an non-fermentable sugar and it inhibits proliferation and growth of Streptococcus mutans [31].

It is recommended to use xylitol in lozenge form as chewing gum is not recommended in patients receiving orthodontic treatment. However, it should be noted that xylitol affects digestive system adversely and overdose usage should be avoided [32].

Sealants, primers, adhesives and other orthodontic materials containing fluoride: Prolongation of orthodontic treatment increases the risk of white spot lesion and caries formation. Therefore, fluoride release from bonding systems around brackets can be useful [10].

Sealants are surface shields that form a protective barrier against acid attacks. There are two types of sealants as chemical and light curing. Chemical-curing sealants were used initially, however disadvantages such as uncured layers, have arisen over time [33]. Studies showed that light-curing sealants are better polymerized and have a better barrier for demineralization as they cover surface completely [33].

Glass ionomer cement is a material that has desired properties such as fluoride release and chemical bonding. However, bonding with glass ionomer increases plaque accumulation around the brackets [3]. Additionally, it is not used for bonding brackets due to low bonding strength. Resin-modified glass ionomer cements were developed to overcome these side effects by adding resin particles in the cement. This adhesive system releases fluoride like conventional glass ionomer cements and has a higher adhesion force [34]. 
It has been thought that fluoride should be added into adhesives for continuous fluoride release, in order to prevent white spot lesion formation during orthodontic treatment without patient co-operation. It was reported that fluoride releasing adhesives provided protection for an area of $1 \mathrm{~mm}$ around brackets, whereas fluoride-free adhesives cannot prevent demineralization around and under brackets [12].

\section{Treatment of white spot lesions}

Topical fluoride application: Topical fluoride application on lesions is considered as the first step of white spot lesion treatment. Application of fluoride in high concentration following completion of orthodontic treatment provides remineralization on lesion surfaces. However untreated parts may remain in deeper layers of lesions and continue to create aesthetic problems. Therefore, penetration of low doses of calcium and fluoride from saliva should be allowed after orthodontic treatment to obtain more aesthetic results [3]. Acid etching followed by fluoride application or acid involving fluoride application facilitates remineralization [17].

Dental bleaching: Bleaching can be applied to camouflage remaining white spot lesions, following natural remineralization which occurs by itself without any intervention. This method can be applied overnight at home or by a professional in dental office by using gel bleaching systems involving different dosages of hydrogen peroxide with the help of transparent trays in patients suffering from yellowish discoloration [35].

Knösel, et al. [36] examined the effect of bleaching on inactive white spot lesions and intact enamel surrounding them following orthodontic treatment. They observed a distinct color change in both white spot lesion area and intact enamel. It was reported that areas with white spot lesions were camouflaged since greater amount of whitening occurred in intact enamel area.

Casein phosphopeptide-amorf calcium phosphate (CPP-ACP) application: Reynolds and Black [23] found that CPP-ACP obtained from milk affected caries development. Free calcium and phosphate ions in CPP-ACP can be easily transferred to enamel surface. CPP-ACP agents are produced in various ways such as foam, mouthwash, topical paste, chewing gum and unsweetened lozenge form to transport calcium and phosphate.

Reynolds [37] reported that using a solution containing 1\% CPP-ACP increased calcium level by $144 \%$ and phosphate level by $160 \%$ in oral environment, which led to decrease in mineral loss by $51 \%$ due to consumption of sugary solution. As a result, the anticaries characteristic of CPP-ACP was demonstrated.

CPP-ACP increases amount of calcium and phosphate ions above the critical level required for remineralization. Thus, it exhibits anticariogenic effect [38].
Increased calcium and phosphate levels in plaque increase $\mathrm{pH}$ level of environment. Thus, demineralization is reduced and remineralization is increased. In a study, a solution containing $1 \%$ CPP-ACP was shown to treat white spot lesions $55 \%$ more compared to a water-only solution [25].

Microabrasion: Microabrasion is a method based on controlled removal of enamel surface by applying different mixtures [39]. As the outer surface of enamel is rich in fluoride and more resistant to external factors, less enamel can be removed in the first step of microabrasion.

The most common method of microabrasion to remove white spot lesions is polishing labial/buccal surface of teeth with a rotating device by using a gel formed mixture of $18 \%$ hydrochloric acid $(\mathrm{HCl})$ and medium grained pumice [40].

Microabrasion process removes some amount of enamel from tooth surface and forms a smoother enamel texture. Calcium and phosphate minerals seals interprismatic spaces as a result of microabrasion and enamel surface becomes more resistant to external factors [41].

Resin infiltration technique: Studies showed that application of fissure sealants to occlusal surfaces prevents caries formation. This fact led clinicians to use of flowable resins for treatment of initial enamel caries [42]. Histological studies showed that microporosity increases in different layers of early enamel lesions. It is known that these porous spaces can absorb liquids such as water like a sponge. In addition, these small porous openings and enlarged intercrystalline spaces act like diffusion paths for acids and dissolved minerals. Filling these porous spaces with low-viscosity resins, instead of completely removing newly emerging lesions, reduces the microporous structure of enamel and supports dental tissue mechanically [43].

Unlike fissure sealant technique, resins applied in caries filling technique do not cover the initial enamel caries like a hat; they rather create a barrier in the lesion by capillary movement. Thus, dental tissues are supported mechanically, whereas fracture and cavitation of surface enamel is prevented. The high penetration value of resin fillers allows enamel lesion to act like a sponge by absorbing the resin and filling pores. It is accepted that preventing spread of organic acids in an enamel lesion prevents progress of decay [44].

Infiltrants are light-curing resin materials developed in a structure that can easily penetrate into the capillary configuration of enamel lesions. The viscosity of these materials is low, their contact angles to enamel are narrow and their surface tensions are high [45].

The basic principle of the resin infiltration technique is; inhibition of lesion progression by blockage of micropores that provide a diffusion pathway for acids 
by using resin [46]. It is suggested that bacteria stuck under the uppermost layer may trigger decay process again despite resin blockage. However some authors suggested that these bacteria are not harmful if the porous openings are sealed properly [47].

Based on the results of in-vivo and in-vitro studies examining the efficacy low-viscosity resins used for filling caries lesions by infiltration, the material named ICON (DMG Chemisch-Pharmazeutische, Hamburg, Germany) has begun to be used. ICON is used effectively in treatment of white spot lesions $[42,43]$.

Invasive therapies are rarely preferred in early enamel lesions that are not cavitated enough to be visible. On the other hand, resin infiltrants can only fill microcavities. Hence, they are not appropriate for treatment of lesions exceeding the superficial $1 / 3$ of dentin and that have visible cavitation. Progression of enamel lesions is recommended to be stopped at an early stage and resin infiltration technique is an effective method as it slows or prevents the progression of lesions [48].

\section{Conclusion}

The exclusion criteria used in this review was not very strict and allowed inclusion of studies having mixed-method study designs. This situation might have caused contradictions in interpretation of some study outcomes and is the limitation of this review.

Oral hygiene motivation, topical fluoride agents, casein phosphopeptide-amorf calcium phosphate agents, antimicrobial agents, tooth bleaching, microabrasion and resin infiltration are the current options for prevention and treatment of white enamel lesions. Reducing the risk of lesion formation by using these methods and early treatment with the appropriate technique is recommended to obtain healthier and more aesthetic results. Future innovations in this field may bring up more treatment options of white spot lesions.

\section{References}

1. Gorelick L, Geiger AM, Gwinnett AJ (1982) Incidence of white spot formation after bonding and banding. Am J Orthod 81: 93-98.

2. Summitt JB, Robbins JW, Hilton TJ, Schwartz RS (2006) Fundamentals of operative dentistry: A contemporary approach. Quintessence Publ Co, Batavia, USA.

3. Bishara SE, Ostby AW (2008) White spot lesions: Formation, prevention, and treatment. Semin Orthod 14: 174-182.

4. Ogaard B (2008) White spot lesions during orthodontic treatment: Mechanisms and fluoride preventive aspects. Semin Orthod 14: 183-193.

5. Curzon ME, Losee FL (1977) Strontium content of enamel and dental caries. Caries Res 11: 321-326.

6. Benson P (2008) Evaluation of white spot lesions on teeth with orthodontic brackets. Semin Orthod 14: 200-208.

7. Zero DT (1995) In Situ caries models. Adv Dent Res 9: 214-
230.

8. Robinson C, Shore RC, Brookes SJ, Strafford S, Wood SR, et al. (2000) The chemistry of enamel caries. Crit Rev Oral Biol Med 11: 481-495.

9. Benson PE, Shah AA, Millett DT, Dyer F, Parkin N, et al. (2005) Fluorides, orthodontics and demineralization: A systematic review. J Orthod 32: 102-114.

10. Newbrun E (1989) Effectiveness of water fluoridation. J Public Health Dent 49: 279-289.

11. van der Veen MH, Mattousch T, Boersma JG (2007) Longitudinal development of caries lesions after orthodontic treatment evaluated by quantitative light-induced fluorescence. Am J Orthod Dentofac Orthop 131: 223-228.

12. Mitchell $L$ (1992) An investigation into the effect of a fluoride releasing adhesive on the prevalence of enamel surface changes associated with directly bonded orthodontic attachments. Br J Orthod 19: 207-214.

13. Koulourides T (1990) Summary of session II: Fluoride and the caries process. J Dent Res 69: 558-558.

14. Chow LC (1990) Tooth-bound fluoride and dental caries. J Dent Res 69: 595-600.

15. Bowden GH (1990) Effects of fluoride on the microbial ecology of dental plaque. J Dent Res 69: 653-659.

16. Gelhard TB, Arends J (1984) Microradiography of in vivo remineralized lesions in human enamel II. J Biol Buccale 12: $59-65$.

17. Linton JL (1996) Quantitative measurements of remineralization of incipient caries. Am J Orthod Dentofacial Orthop 110: 590-597.

18. Willmot DR (2004) White lesions after orthodontic treatment: Does low fluoride make a difference? J Orthod 31: 235-242.

19. Institute of medicine (1997) Dietary reference intakes for calcium, phosphorus, magnesium, vitamin $\mathrm{d}$, and fluoride. National Academies Press. Washington, USA, 432.

20. Heller KE, Eklund SA, Burt BA (1997) Dental caries and dental fluorosis at varying water fluoride concentrations. J Public Health Dent 57: 136-143.

21. ten Cate JM, Duijsters PP (1983) Influence of fluoride in solution on tooth demineralization II. Microradiographic data. Caries Res 17: 513-519.

22. Harper DS, Osborn JC, Hefferren JJ, Clayton R (1986) Cariostatic evaluation of cheeses with diverse physical and compositional characteristics. Caries Res 20: 123-130.

23. Reynolds EC, Black CL (1987) Reduction of chocolate's cariogenicity by supplementation with sodium caseinate. Caries Res 21: 445-451.

24. Schüpbach P, Neeser JR, Golliard M, RouvetM, Guggenheim B (1996) Incorporation of caseinoglycomacropeptide and caseinophosphopeptide into the salivary pellicle inhibits adherence of mutans streptococci. J Dent Res 75: 17791788.

25. Reynolds EC, Cain CJ, Webber FL, Black CL, Riley PF, et al. (1995) Anticariogenicity of calcium phosphate complexes of tryptic casein phosphopeptides in the rat. $J$ Dent Res 74: 1272-1279.

26. Riordan PJ (1993) Dental fluorosis, dental caries and fluoride exposure among 7-year-olds. Caries Res 27: 71-77.

27. Kardos S, Shi B, Sipos T (1999) The in vitro demineralization potential of a sodium fluoride, calcium and phosphate ioncontaining dentifrice under various experimental conditions. J Clin Dent 10: 22-25. 
28. Beyth N, Redlich M, Harari D, Friedman M, Steinberg D (2003) Effect of sustained-release chlorhexidine varnish on streptococcus mutans and actinomyces viscosus in orthodontic patients. Am J Orthod Dentofac Orthop 123: 345-348.

29. Madléna M, Vitalyos G, Márton S, Nagy G (2000) Effect of chlorhexidine varnish on bacterial levels in plaque and saliva during orthodontic treatment. J Clin Dent 11: 42-46.

30. Gehlen I, Netuschil L, Georg T, Reich E, Berg R, et al. (2000) The influence of a $0.2 \%$ chlorhexidine mouthrinse on plaque regrowth in orthodontic patients. A randomized prospective study. Part II: Bacteriological parameters. J Orofac 61: 138-148.

31. Hanham A, Addy M (2001) The effect of chewing sugar-free gum on plaque regrowth at smooth and occlusal surfaces. J Clin Periodontol 28: 255-257.

32. Sengun A, Sari Z, Ramoglu SI, Malkoç S, Duran I (2004) Evaluation of the dental plaque $\mathrm{pH}$ recovery effect of a xylitol lozenge on patients with fixed orthodontic appliances. Angle Orthod 74: 240-244.

33. Joseph VP, Rossouw PE, Basson NJ (1994) Some "sealants" seal-a scanning electron microscopy (SEM) investigation. Am J Orthod Dentofac Orthop 105: 362-368.

34. Diaz-Arnold AM, Holmes DC, Wistrom DW, Swift EJ (1995) Short-term fluoride release/uptake of glass ionomer restoratives. Dent Mater 11: 96-101.

35. Donly KJ, Gerlach RW (2002) Clinical trials on the use of whitening strips in children and adolescents. Gen Dent 50: 242-245.

36. Knösel M, Attin R, Becker K, Attin T (2007) External bleaching effect on the color and luminosity of inactive white-spot lesions after fixed orthodontic appliances. Angle Orthod 77: 646-652.

37. Reynolds EC (1998) Anticariogenic complexes of amorphous calcium phosphate stabilized by casein phosphopeptides: A review. Spec Care Dentist 18: 8-16.
38. Reynolds EC (1997) Remineralization of enamel subsurface lesions by casein phosphopeptide-stabilized calcium phosphate solutions. J Dent Res 76: 1587-1595.

39. Rodd HD, Davidson LE (1997) The aesthetic management of severe dental fluorosis in the young patient. Dent Update 24: 408-411.

40. Welbury RR, Carter NE (1993) The hydrochloric acidpumice microabrasion technique in the treatment of postorthodontic decalcification. Br J Orthod 20: 181-185.

41. Donly KJ, O’Neill M, Croll TP (1992) Enamel microabrasion: A microscopic evaluation of the "abrosion effect". Quintessence Int 23: 175-179.

42. Paris S, Meyer-Lueckel H (2009) Masking of labial enamel white spot lesions by resin infiltration-a clinical report. Quintessence Int 40: 713-718.

43. Kielbassa AM, Muller J, Gernhardt CR (2009) Closing the gap between oral hygiene and minimally invasive dentistry: A review on the resin infiltration technique of incipient (proximal) enamel lesions. Quintessence Int 40: 663-681.

44. Kugel G, Arsenault P, Papas A (2009) Treatment modalities for caries management, including a new resin infiltration system. Compend Contin Educ Dent 3: 1-10.

45. Paris S, Meyer-Lueckel H, Cölfen H, Kielbassa AM (2007) Penetration coefficients of commercially available and experimental composites intended to infiltrate enamel carious lesions. Dent Mater 23: 742-748.

46. Meyer-Lueckel H, Paris S (2008) Progression of artificial enamel caries lesions after infiltration with experimental light curing resins. Caries Res 42: 117-124.

47. Kidd EA (2004) How "clean" must a cavity be before restoration? Caries Res 38: 305-313.

48. Paris S, Meyer-Lueckel H, Kielbassa AM (2007) Resin infiltration of natural caries lesions. J Dent Res 86: 662-666. 